\title{
Avaliação de Resultados da Assistência em Saúde Mental por Usuários
}

\section{Evaluation of Mental Health Care Results by Users}

\author{
Mônica Silva de Bessa ${ }^{1}$ \\ João de Deus de Araújo Filho ${ }^{1}$ \\ Dulcian Medeiros de Azevedo ${ }^{2}$ \\ Marina de Góes Salvetti ${ }^{3}$ \\ Gilson de Vasconcelos Torres ${ }^{4}$
}

\section{RESUMO}

Objetivo: avaliar os resultados da assistência em saúde de um centro de atenção psicossocial (CAPS). Metodologia: estudo avaliativo, com delineamento não experimental, realizado num CAPS I do oeste potiguar, entre os meses de março e abril de 2014. Foram entrevistados 32 usuários do serviço, com aplicação de instrumento de pesquisa desenvolvido pelos autores, a partir de quatro domínios: autonomia, relacionamento, volição e crises. Dados analisados por meio de estatística descritiva. Resultados: Os usuários predominantes eram homens (56,3\%), com idade entre 34 e 43 anos (40\%), casados $(87,5 \%)$, ensino fundamental I incompleto $(50 \%)$, moradores da zona urbana (65\%). O transtorno de humor e/ou afetivo foi o diagnóstico principal, sendo $57,1 \%$ entre as mulheres. Recebiam tratamento entre sete e oito anos no CAPS pesquisado (49,3\%), compareciam ao serviço duas vezes por semana $(46,5 \%)$. Referiram internamento hospitalar a menos de um ano $(9,4 \%)$. Os domínios para avaliação dos resultados da assistência obtiveram melhoras após o tratamento no CAPS, com destaque para "Crises" $(78,1 \%)$ e "Relacionamento" $(71,9 \%)$. A avaliação de serviços de saúde mental por usuários é um mecanismo que pode contribuir para sua autonomia, gestão do cuidado e do serviço. Conclusão: Os resultados da assistência em saúde foram positivos em todos os domínios avaliados. O serviço pesquisado possui fragilidades no tocante ao fluxo de atendimento do usuário, participação familiar, condução da agenda terapêutica, implementação de projeto terapêutico singular e ampliação de atividades extramuros.

DESCRITORES: Avaliação de Serviços de Saúde. Avaliação de Resultados da Assistência ao Paciente. Serviços de Saúde Mental. Saúde Mental.

\begin{abstract}
Objective: To evaluate the health care results of a psychosocial care center (CAPS). Methodology: It is an evaluative study, with a non-experimental design, which was performed in a unity of the CAPS I in the west region of Rio Grande do Norte, between March and April 2014. We conducted interviews with 32 users of this service by applying a research instrument developed by the authors, based on four domains: autonomy, relationship, volition and crises. Data were analyzed through descriptive statistics. Results: Users were predominantly male $(56.3 \%)$, aged between 34 and 43 years (40\%), married $(87.5 \%)$, with incomplete elementary school I (50\%) and lived in the urban area $(65 \%)$. The mood and/or affective disorder was the main diagnosis, with $57.1 \%$ among female participants. They received treatment between seven and eight years in the CAPS $(49.3 \%)$ and attended the service twice a week $(46.5 \%)$. Only $9.4 \%$ mentioned admission less than a year ago $(9.4 \%)$. The domains for evaluating the care results achieved improvements after the treatment in the CAPS, with emphasis being placed on the "Crises" $(78.1 \%)$ and "Relationship" $(71.9 \%)$ domains. The evaluation of mental health services by users is a mechanism that can contribute to their autonomy, as well as to the management of care and service. Conclusion: The health care results were positive in all evaluated domains. The surveyed service has weaknesses regarding the flow of user care, family involvement, conduction of the therapeutic program, implementation of a unique therapeutic project, and expansion of activities outside the walls.
\end{abstract}

DESCRIPTORS: Health Services Evaluation. Patient Outcome Assessment. Mental Health Services. Mental Health.

- Enfermeira. Caicó-RN.

2- Professor do Curso de Graduação em Enfermagem (UERN/Campus Caicó, Caicó-RN

3- Professor da Escola de Enfermagem da USP (EEUSP), São Paulo-SP.

4- Professor do Departamento de Enfermagem, Universidade Federal do Rio Grande do Norte (UFRN/Campus Central). Natal-RN 
A Reforma Psiquiátrica (RP) brasileira surge em favor de mudanças do modelo hospitalocêntrico, para um outro substitutivo de caráter comunitário e aberto. É contemporânea do movimento sanitário, que pedia a alteração dos modelos de atenção e gestão nas práticas de saúde, a defesa da saúde coletiva, o protagonismo dos trabalhadores e usuários dos serviços de saúde nos processos de gestão e produção de tecnologias de cuidado ${ }^{1}$.

Passadas duas décadas, a RP tem no Centro de Atenção Psicossocial (CAPS) o pilar central de atendimento em saúde mental comunitário, visando oferecer atendimento com a intencionalidade de reabilitação e reinserção social de pessoas com transtorno mental ou usuária de álcool e outras drogas ${ }^{2}$. Representa um lugar de referência e de cuidado, que pretende garantir o exercício da cidadania e a inclusão social de usuários e seus familiares ${ }^{3}$.

Apesar dos avanços da RP brasileira, ainda existem obstáculos e fragilidades na aplicação dos princípios reformistas, como a dificuldade na articulação e diálogo entre CAPS e atenção básica, serviços de urgência/emergência insuficientes, profissionais da área despreparados. Além disso, persistem ou ressurgem formas de apego a práticas manicomiais, imersas nos serviços substitutivos ${ }^{4}$.

Estudos avaliativos proporcionam um olhar diferenciado que levam a questionamentos e pensamentos de modificações no serviço/atendimento prestado ao usuário. Com as mudanças advindas da RP, a avaliação dos serviços de saúde mental é uma necessidade real, sobretudo para o devido acompanhamento da política nacional de saúde ${ }^{5}$.

Propostas avaliativas são consideravelmente pertinentes diante de tais obstáculos, promovendo avaliação do desempenho dos sistemas de serviços da saúde, com objetivo de encontrar a qualidade do serviço e da gestão ${ }^{5}$. O estudo objetivou avaliar os resultados da assistência em saúde de um CAPS, na perspectiva de usuários do serviço.

\section{MATERIAL E MÉTODOS}

Estudo avaliativo, com delineamento não experimental, realizado no CAPS I, da região oeste potiguar, entre os meses de março e abril de 2014. Fundado há dez anos, o CAPS pesquisado concebe uma alternativa de tratamento substitutiva até então inexistente para o município e a região. Anteriormente, as demandas de saúde mental eram referenciadas a um hospital psiquiátrico distante 194 km. Dados do Ministério da Saúde apontam 1.069 CAPS I, exercendo papel fundamental na municipalização das ações de saúde mental nos pequenos municípios do país. Destes, 17 CAPS I estão no Rio Grande do Norte ${ }^{6}$.

Além de prestar atendimento a usuários com transtornos mentais graves e persistentes, o CAPS também funciona como ambulatório especializado de saúde mental, onde o psiquiatra do serviço atende todas as demandas de saúde mental do município. Tal fato explica os quase 150 usuários cadastrados, apesar de, no momento da coleta, existirem 46 em participação efetiva das atividades terapêuticas ofertadas, com média de 15 usuários/dia.

Foram entrevistados 32 usuários e utilizado o instrumento "Escala Avaliação dos Resultados da Assistência em Saúde no CAPS/Usuários e Familiares" (EARAS-CAPS), que possui dez assertivas e quatro domínios avaliativos, com três alternativas de resposta ("piorou", "não mudou" e "melhorou").

A EARAS-CAPS recebeu validação de conteúdo de 50 juízes de diferentes regiões do país, com formação e atuação no campo da saúde mental, através de duas fases do Método Delfh (85\% de retorno entre as fases). Além deste instrumento, os pesquisadores se valeram da observação participante (40 horas) para coletar outros dados relacionados à gestão do serviço e do cuidado, com auxílio de um diário de campo.

Todos os participantes preencheram o critério de inclusão, que era estar em tratamento semi-intensivo ou não intensivo no CAPS, há pelo menos um ano, e assinaram o Termo de Consentimento Livre e Esclarecido (TCLE), conforme recomendação da Resolução CNS Nº. 466/127. A pesquisa possui parecer ético favorável $\left(n^{\circ}\right.$. 292.786) do Comitê de Ética em Pesquisa, do Hospital Universitário Onofre Lopes (CEP/HUOL/ UFRN - CAAE: 12288313.8.0000.5292).

Os dados foram processados por meio do Software Statistical Packagefor the Social Sciences (SPSS), e analisados mediante estatística descritiva, apresentados em tabelas.

\section{RESULTADOS}

A Tabela 1 apresenta a caracterização dos usuários segundo variáveis socioculturais.

A Tabela 2 traz os dados sobre variáveis socioeconômicas e diagnóstico psiquiátrico.

As características clínicas e aquelas relacionadas ao tratamento dos usuários estão apresentadas na Tabela 3.

A Tabela 4 traz os resultados das variáveis relacionadas aos Resultados com o tratamento no CAPS. 
Tabela 1. Distribuição absoluta e percentual dos usuários do CAPS segundo sexo, idade, estado civil, escolaridade, zona de moradia e convivência. Oeste Potiguar, 2014.

\begin{tabular}{|c|c|c|c|c|c|}
\hline & & $\mathrm{n}$ & $\%$ & $\mathrm{n}$ & $\%$ \\
\hline \multirow{2}{*}{\multicolumn{2}{|c|}{ Sexo }} & \multicolumn{2}{|c|}{ Feminino } & \multicolumn{2}{|c|}{ Masculino } \\
\hline & & 14 & 43,8 & 18 & 56,3 \\
\hline \multicolumn{6}{|l|}{ Idade } \\
\hline 27 a 33 & & 4 & 28,6 & 2 & 11,1 \\
\hline 34 a 43 & & 4 & 28,6 & 9 & 50,0 \\
\hline 44 a 53 & & 4 & 28,6 & 6 & 33,3 \\
\hline 54 a 60 & & 2 & 14,2 & 1 & 5,6 \\
\hline \multicolumn{6}{|l|}{ Estado Civil } \\
\hline Casado & & 9 & 64,3 & 12 & 66,7 \\
\hline Solteiro & & 4 & 28,6 & 5 & 27,8 \\
\hline Separado & & 0 & 0,0 & 1 & 5,6 \\
\hline Viúvo & & 1 & 7,1 & 0 & 0,0 \\
\hline \multicolumn{6}{|l|}{ Escolaridade } \\
\hline Analfabeto & & 3 & 21,4 & 5 & 27,8 \\
\hline $\begin{array}{l}\text { Fundamental } \\
\text { Incompleto }\end{array}$ & I & 7 & 50,0 & 9 & 50,0 \\
\hline $\begin{array}{l}\text { Fundamental } \\
\text { Completo }\end{array}$ & I & 3 & 21,4 & 2 & 11,1 \\
\hline Médio Completo & & 0 & 0,0 & 2 & 11,1 \\
\hline Superior incompleto & & 1 & 7,1 & 0 & 0,0 \\
\hline \multicolumn{6}{|l|}{ Zona de Moradia } \\
\hline Urbana & & 9 & 64,3 & 12 & 66,7 \\
\hline Rural & & 5 & 35,7 & 6 & 33,3 \\
\hline \multicolumn{6}{|l|}{ Convivência } \\
\hline Cônjuge e Filhos & & 4 & 28,6 & 9 & 50,0 \\
\hline Pais e Filhos & & 4 & 28,6 & 3 & 16,7 \\
\hline Cônjuge & & 4 & 28,6 & 2 & 11,1 \\
\hline $\begin{array}{l}\text { Pais, Irmão } \\
\text { Sobrinho }\end{array}$ & $e$ & 1 & 7,1 & 2 & 11,1 \\
\hline Outros & & 1 & 7,1 & 2 & 11,1 \\
\hline
\end{tabular}


Tabela 2: Distribuição absoluta e percentual dos usuários do CAPS segundo sexo, profissão, tipo de renda e diagnóstico psiquiátrico. Oeste Potiguar, 2014.

\begin{tabular}{|c|c|c|c|c|}
\hline & $n$ & $\%$ & $\mathrm{n}$ & $\%$ \\
\hline Sexo & \multicolumn{2}{|c|}{ Feminino } & \multicolumn{2}{|c|}{ Masculino } \\
\hline & 14 & 43,8 & 18 & 56,3 \\
\hline Profissão & & & & \\
\hline Agricultor & 10 & 71,4 & 13 & 72,2 \\
\hline Nenhuma & 2 & 14,3 & 2 & 11,1 \\
\hline Vendedor & 0 & 0,0 & 1 & 5,6 \\
\hline Do lar & 1 & 7,1 & 0 & 0,0 \\
\hline Atendente & 1 & 7,1 & 0 & 0,0 \\
\hline Técnico de & 0 & 0,0 & 1 & 5,6 \\
\hline Enfermagem & & & & \\
\hline Carregador & 0 & 0,0 & 1 & 5,6 \\
\hline Total & 14 & 100,0 & 18 & 100,0 \\
\hline \multicolumn{5}{|l|}{ Tipo de Renda } \\
\hline $\mathrm{BPC}$ & 8 & 57,1 & 11 & 61,1 \\
\hline Nenhuma & 4 & 28,6 & 4 & 22,2 \\
\hline Bolsa Família & 1 & 7,1 & 1 & 5,6 \\
\hline Trabalho Informal & 1 & 7,1 & 0 & 0,0 \\
\hline Outros & 0 & 0,0 & 2 & 11,2 \\
\hline \multicolumn{5}{|l|}{$\begin{array}{l}\text { Diagnóstico } \\
\text { Psiquiátrico }\end{array}$} \\
\hline F.30/391 & 8 & 57,1 & 5 & 27,8 \\
\hline F. $20 / 29^{2}$ & 2 & 14,3 & 5 & 27,8 \\
\hline F.40/48 3 & 3 & 21,4 & 3 & 16,7 \\
\hline F.06 & 0 & 0,0 & 2 & 11,1 \\
\hline F.70/795 & 1 & 7,1 & 1 & 5,6 \\
\hline G. $40^{* * * * * *}$ & 0 & 0,0 & 2 & 11,1 \\
\hline
\end{tabular}

${ }^{1}$ Transtornos do Humor/Afetivos

${ }^{2}$ Esquizofrenia, Transtornos Esquizotípicos e Transtornos Delirantes

${ }^{3}$ Transtornos Neuróticos, Transtornos Relacionados à Estresse e Transtornos Somatoformes

${ }^{4}$ Outros transtornos mentais especificados devidos à lesão e disfunção cerebral e à doença física

${ }^{5}$ Retardo mental

${ }^{6}$ Epilepsia 
Tabela 3 - Distribuição absoluta e percentual dos usuários, segundo a participação no CAPS e internamento psiquiátrico. Oeste Potiguar, 2014.

\begin{tabular}{|c|c|c|}
\hline Tempo de tratamento (anos) & $\mathrm{n}$ & $\%$ \\
\hline $1-2$ & 7 & 22,6 \\
\hline $3-4$ & 4 & 13,5 \\
\hline $5-6$ & 5 & 14,6 \\
\hline $7-8$ & 16 & 49,3 \\
\hline \multicolumn{3}{|l|}{$\begin{array}{l}\text { Frequência de Participação } \\
\text { Semanal }\end{array}$} \\
\hline Uma vez & 6 & 19,8 \\
\hline Duas vezes & 15 & 46,5 \\
\hline Três a Cinco vezes & 11 & 33,7 \\
\hline \multicolumn{3}{|l|}{ Atividades no CAPS } \\
\hline Acolhimento e Oficina de Arte & 18 & 56,4 \\
\hline Acolhimento, Oficina de Arte e Coral & 5 & 14,8 \\
\hline $\begin{array}{l}\text { Acolhimento, Oficina de Arte e } \\
\text { Esporte }\end{array}$ & 3 & 9,6 \\
\hline Acolhimento & 3 & 9,6 \\
\hline Outros & 3 & 9,6 \\
\hline \multicolumn{3}{|l|}{$\begin{array}{l}\text { Não internamento psiquiátrico } \\
\text { (anos) }\end{array}$} \\
\hline Nunca & 19 & 59,2 \\
\hline $1-5$ & 6 & 18,8 \\
\hline $6-10$ & 6 & 18,8 \\
\hline $11-30$ & 1 & 3,2 \\
\hline \multicolumn{3}{|l|}{ Internamento a menos de um ano } \\
\hline Não & 29 & 90,6 \\
\hline Sim & 3 & 9,4 \\
\hline
\end{tabular}

Tabela 4: Distribuição percentual dos Domínios relacionados a Resultados com o tratamento no CAPS, segundo usuários. Oeste Potiguar, 2014.

\begin{tabular}{l|c|c|c}
\hline Domínios EARAS-CAPS & Piorou & $\begin{array}{c}\text { Não } \\
\text { Mudou }\end{array}$ & Melhorou \\
\hline Resultados/Autonomia & 9,4 & 23,4 & 67,2 \\
Resultados/Relacionam & 10,4 & 17,7 & 71,9 \\
ento & & & \\
Resultados/Volição & 8,6 & 26,6 & 64,8 \\
Resultados/Crises & 9,4 & 12,5 & 78,1 \\
\hline
\end{tabular}




\section{DISCUSSÃO}

No tocante à caracterização dos participantes, predominaram os homens $(56,3 \%)$, com baixa escolaridade (ensino fundamental incompleto $-50 \%$ ) e moradores da zona urbana $(65,0 \%)$. Considera-se que a profissão de agricultor é majoritária na região Oeste potiguar e no município pesquisado. Noutro estudo, eram homens $(61,4 \%)$, com Ensino Fundamental Completo/Incompleto $(50,0 \%)$ e solteiros $(60,0 \%)^{8}$.

Em relação à renda, a mencionaram o recebimento de Benefício de Prestação Continuada (BPC), algo imprescindível para ajuda e apoio financeiro diante do tratamento e subsistência. $O$ BPC contribui para a manutenção de condições mínimas de vida para pessoas com alguma deficiência que se encontram em situação de pobreza, considerado dispositivo de proteção social, visando a garantia de um valor básico de renda às pessoas consideradas incapacitadas para a vida independente e para o trabalho' .

Os Transtornos do Humor/Afetivos representaram o principal diagnóstico entre os participantes, sobretudo para as mulheres. Entre os homens, houve similaridade entre este diagnóstico de Esquizofrenia, Transtornos Esquizotípicos e Transtornos Delirantes. Semelhante ao estudo realizado em Lorena-SP, onde os diagnósticos de transtornos severos e persistentes (psicoses e esquizofrenias) foram predominantes entre os homens e, nas mulheres, prevaleceram os transtornos do humor e de ansiedade ${ }^{8}$. Noutra pesquisa, nos homens predominaram diagnósticos de transtornos mentais e comportamentais decorrentes do uso abusivo de substâncias psicoativas e, entre as mulheres, esquizofrenia, transtornos esquizotípicos e transtornos delirantes ${ }^{10}$.

A definição do diagnóstico psiquiátrico por si só não cabe na proposta psicossocial, defendida pelo movimento reformista. Quando parte integrante de um plano terapêutico efetivo, torna-se imprescindível para valorizar as particularidades do sujeito e de sua família. Entretanto, no CAPS pesquisado não foi observado execução de um cronograma de atividades terapêuticas, nem de oficinas terapêuticas idealizadas na perspectiva de um projeto terapêutico singular (PTS), representando dois problemas importantes de conduta terapêutica no serviço. O PTS é um conjunto de propostas para atender a condutas terapêuticas do usuário ou de uma família, e surge pela discussão coletiva de uma equipe interdisciplinar na intenção de uma melhor terapêutica/tratamento ${ }^{11}$
Os usuários recebiam tratamento entre sete e oito anos (49,3\%), coincidindo com o tempo de funcionamento do CAPS. A participação se dá com uma frequência de duas vezes semanais $(46,5 \%)$, com predominância de duas atividades: acolhimento e oficina de artes $(56,4 \%)$. O acolhimento mencionado e ofertado no CAPS pesquisado era realizado de forma diária, com a maioria dos profissionais e usuários, numa roda de conversa, dando início às atividades e demandas do dia. A oficina de artes era conduzida pela Artesã e Arte-terapeuta do serviço, ofertando atividades de recorte e colagem, pintura, confecção de tapetes, dentre outros afazeres lúdico-recreativos.

Os usuários pesquisados ainda realizavam atividades de esporte e lazer, principalmente em datas comemorativas locais e nacionais, promovendo bem estar e inserção comunitária externa ao CAPS. Promover o empoderamento dentro da realidade de cada um é o objetivo dessas ações, que utilizam recursos culturais para retirar esses indivíduos e seus familiares do cárcere de suas próprias mentes $^{12}$.

A utilização de oficinas terapêuticas é um excelente instrumento para alcançar diversos fins, e como proposta artística coletiva, estimula a autonomia e o protagonismo social, incentivando o reconhecimento do seu papel no grupo, desenvolvimento da autoestima e aspectos relativos à reinserção e cidadania dos usuários ${ }^{13-15}$.

Os resultados relatados pelos usuários foram, em todas as variáveis, avaliados de forma positiva, possuindo melhora significativa no relacionamento $(71,9 \%)$ e crises $(78,1 \%)$ após o tratamento ofertado pelo CAPS. No município de Pelotas-RS, usuários de um CAPS apresentaram melhoras no relacionamento com amigos $(43,3 \%)$, com familiares $(60,8 \%)$ e outras pessoas $(49,4 \%)$, após tratamento neste serviço ${ }^{16}$.

Os resultados avaliados no instrumento desenvolvido pelos autores nesta pesquisa não pretendem esgotar, contrapor ou substituir outros instrumentos específicos à avaliação do tratamento de usuários de saúde mental. Destaca-se no Brasil o pioneirismo da Escala de Mudança Percebida pelos Pacientes (EMP-Pacientes), desenvolvida por pesquisadores da Universidade Federal São João Del-Rey (UFSJ), em Minas Gerais ${ }^{17}$.

No momento da pesquisa, não foi observado usuários acompanhados por familiares, demonstrando a liberdade e certa autonomia deles no uso do equipamento. Percebe-se que a partir da RP brasileira, familiares já reconhecem ganhos e conquistas, a exemplo de familiares $(74 \%)$ 
que avaliaram como bom o serviço ofertado pelo CAPS $^{18}$. Com a implantação dos serviços substitutivos, especialmente os CAPS, houve redução significativa nas internações por complicações psiquiátricas, refletindo na redução dos leitos de hospitais psiquiátricos ${ }^{19}$.

Esta pesquisa ainda revelou que 59,2\% dos usuários pesquisados nunca passaram por internações psiquiátricas $(59,2 \%)$, elencando o CAPS como principal promotor de cuidado para seu tratamento, algo semelhante ao CAPS II de Recife-PE, onde $57,0 \%$ nunca foram internados em hospital psiquiátrico, antes da admissão no CAPS $^{20}$.

Acredita-se que este serviço se tornou essencial para uma assistência comunitária, com apoio psicossocial e (re)inserção social da realidade pesquisada. Por outro lado, é preciso defender a articulação com outras áreas do conhecimento, políticas públicas e movimentos sociais libertários e democráticos, criando possibilidades de emancipação do sujeito e do núcleo familiar ${ }^{21}$.

Os profissionais que compõem o CAPS têm papel imprescindível junto ao usuário. Durante a pesquisa, observou-se que o Enfermeiro e o Pedagogo eram os profissionais que mais participavam de atividades externas ao CAPS ${ }^{22}$. Efetivar uma assistência multidisciplinar de forma articulada e integrada ${ }^{23}$ é um movimento a ser percorrido, com participação de todos os profissionais no tratamento e promoção de uma terapêutica individual e grupal. Ações alicerçadas no acolhimento, estímulo à convivência social, quebra de estigmas e valorização das potencialidades são mecanismos

\section{REFERÊNCIAS}

1. Ministério da Saúde (BR). Reforma psiquiátrica e política de saúde mental no Brasil. Ministério da Saúde: Brasília; 2005.

2. Kantorski LP, Jardim VMR, Delpino GB, Lima LM, Schwartz E, Heck RM. Perfil dos familiares cuidadores de usuários de centros de atenção psicossocial do sul do Brasil. Rev. gaúcha enferm. 2012; 33(1):85-92.

3. Ministério da Saúde (BR). Manual de estrutura física dos centros de atenção psicossocial e unidades de acolhimento. Ministério da Saúde: Brasília; 2013.

4. Azevedo DM. Avaliação de serviços de saúde: perspectivas atuais da pesquisa em saúde mental. Rev. enferm. UFPE on line. 2011; 5(8):1-3.

5. Azevedo DM, Oliveira AM, Melo GSM, Salvetti MG, Vasconcelos QLDAQ, Torres GV. Avaliação da assistência em saúde mental num centro de atenção psicossocial na perspectiva dos profissionais. Rev. Bras. Pesq. Saúde. 2014; 16(2):109-16. essenciais para substituição do modelo asilar, constituindo uma nova filosofia de trabalho em todos os serviços substitutivos, especialmente nos CAPS ${ }^{24}$.

A avaliação de serviços de saúde mental por usuários é um mecanismo que pode contribuir para autonomia, enquanto promotor e sujeito ativo do seu tratamento, para gestão do cuidado e do serviço. Destaca-se ainda o relacionamento do usuário com o meio social e familiar para a implementação e fortalecimento do tratamento, principalmente, quando se tem uma nova política de assistência à saúde, aberta e comunitária, permitindo a livre convivência e trânsito no ambiente terapêutico.

Esta pesquisa admite limitações no tocante à quantidade de sujeitos avaliados, além do número de serviços (um CAPS). Entretanto, defende-se que tais circunstâncias não invalidam os resultados alcançados, suscitando a divulgação e estímulo ao processo avaliativo no cenário dos serviços de saúde públicos, sobretudo em saúde mental a partir da RP em curso.

\section{CONCLUSÃO}

Os resultados da assistência à saúde foram avaliados de forma positiva por todos os usuários. Entretanto, o serviço ainda possui fragilidades nas abordagens iniciais dos usuários (fluxo de atendimento), participação familiar, condução da agenda terapêutica, implementação de PTS e ampliação de atividades extramuros.
6. Ministério da Saúde (BR). Saúde Mental em Dados 12 Ministério da Saúde: Brasília; 2015.

7. Ministério da Saúde (BR). Conselho Nacional de Saúde. Resolução 466, de 12 de dezembro de 2012: diretrizes e normas regulamentadoras de pesquisa envolvendo seres humanos. Ministério da Saúde: Brasília; 2012.

8. Pereira MO, Souza JM, Costa ÂM, Vargas D, Oliveira MAF, Moura WN. Perfil dos usuários de serviços de saúde mental do município de Lorena - São Paulo. Acta Paul Enferm. 2012; 25(1):48-54.

9. Nogueira KER. Novo modelo de avaliação para o acesso ao BPC da política de assistência social: o olhar das assistentes sociais da gerência executiva do INSS Fortaleza [Dissertação de Mestrado]. Fortaleza: Universidade Estadual do Ceará; 2011. 130 p. Disponível em: <http:// www.uece.br/politicasuece/dmdocuments/karla_emanuela rocha.pdf>. Acesso em: 13 out. 2015.

10. Pelisoli CL, Moreira ÂK. Caracterização epidemiológica dos usuários do centro de atenção psicossocial Casa Aberta. Rev. Psiquiatr. 2005; 27(3):270-77. 
11. Ministério da Saúde (BR). Clínica ampliada, equipe de referência e projeto terapêutico singular. Ministério da Saúde: Brasília; 2008.

12. Azevedo EB, Ferreira Filha MO, Araruna MHM, Carvalho RN, Cordeiro RC, Silva VCL. Práticas inclusivas extramuros de um centro de atenção psicossocial: possibilidades inovadoras. Saúde em debate. 2012; 36(95):595-605.

13. Lima FP, Oliveira ML, Wilrich JQ. Relato de experiência-a arte como instrumento de reinserção social. J Nurs Health. 2012; 2suppl:S265-75.

14. Andrade LA, Lima ICRL, Velôso TMG. A utilização de recursos audiovisuais em oficinas com usuários de um centro de atenção psicossocial (CAPS). Cad. Bras. Saúde Mental. 2016; 8(18):132-45.

15. Pammi PV, Tomasi E. "Uma família que trabalha": oficinas de geração de trabalho e renda da reabilitação, trabalho e arte de Pelotas (RS). Outra Economia. 2013; 7(12):100-08.

16. Santos ZD, Munhoz TN. A mudança percebida pelo usuário do centro de atenção psicossocial frente ao tratamento. Rev. Bras. Pesq. Saúde. 2015; 17(2):22-27.

17. Bandeira MB, Andrade MCR, Costa CS, Silva MA. Percepção dos pacientes sobre o tratamento em serviços de saúde mental: validação da Escala de Mudança Percebida. Psicologia: reflexão e crítica. 2010; 24(2):236-44.

18. Azevedo DM, Oliveira AM, Freitas CCS, Araújo RO, Melo GSM, Torres GV. Qualidade da assistência no centro de atenção psicossocial: perspectiva de familiares. Rev. Bras. Pesq. Saúde. 2014; 16(4):6-12.

19. Carvalho MDA, Silva HO, Rodrigues LV. Perfil epidemiológico dos usuários da rede de saúde mental do município de Iguatu, CE. Rev. eletrônica saúde mental álcool drog. 2010; 6(2):337-49.
20. Paula CTC. Perfil epidemiológico dos usuários de um centro de atenção psicossocial na cidade de Recife. Cad. Bras. Saúde Mental. 2010; 2(4-5):94-105.

21. Silva ATMC, Amarante P, Gomes ALC, Braga JEF, Valença AMG, Padilha WWN. A propósito da diversidade, cultura e saúde mental: novas dimensões para a compreensão da loucura. Rev. bras. ciênc. saúde. 2012; 16(3):435-38.

22. Araújo Filho JD, Bessa MS, Azevedo DM. Assistência de saúde mental em um centro de atenção psicossocial: avaliação na perspectiva profissional. J Nurs Health. 2016; 6(2):279-86.

23. Clementino FS, Miranda FAN, Martiniano CS, Marcolino EC, Pessoa Júnior JM, Dias JA. Avaliação da estrutura organizacional dos centros de atenção psicossocial do município de Campina Grande, Paraíba. Rev. bras. ciênc. saúde. 2016; 20(4):261-68

24. Azevedo DM, Oliveira AM, Melo GSM, Salvetti MG, Vasconcelos QLDAQ, Torres GV. Avaliação da assistência em saúde num centro de atenção psicossocial na perspectiva dos profissionais. Rev. bras. pesq. saúde. 2014; 16(2):109-16

\section{CORRESPONDÊNCIA}

Dulcian Medeiros de Azevedo

Av. Rio Branco, $n^{\circ} 725$. Bairro Paraíba.

Caicó - RN - Brasil - CEP: 59300-000

E-mail: professordulcian@gmail.com 